\title{
A INSERÇÃO DO ENSINO DE CUSTOS NA DISCIPLINA ADMINISTRAÇÃO APLICADA À ENFERMAGEM
}

INSERTION OF COST TEACHING IN THE DISCIPLINE OF ADMINISTRATION APPLIED TO NURSING

\author{
LA INSERCIÓN DE LA ENSEÑANZA DE COSTOS EN LA DISCIPLINAADMINISTRACIÓN \\ APLICADAALAENFERMERÍA
}

Ivone Maria Fonseca Francisco', Valéria Castilho²

\section{RESUMO}

O objetivo deste texto foi realizar um resgate teórico sobre a trajetória da disciplina de Administração Aplicada à Enfermagem, na graduação, ao longo da história do ensino da Enfermagem Brasileira, relacionando-a ao ensino de Custos. Percebemos, inseridas no ensino dessa disciplina, diversas citações diretas referentes ao tema Custos ou Economia em Saúde, desde o primeiro currículo de Enfermagem de 1890 até as Diretrizes Curriculares Nacionais do Curso de Graduação em Enfermagem de 2001, além de citações indiretas relacionadas ao gerenciamento, tomada de decisão, assessorias em projetos de saúde, entre outras, mas sem haver uma continuidade.

\section{DESCRITORES}

Enfermagem (organização e administração).

Custos e análise de custo. Ensino.

\section{ABSTRACT}

The objective of this text was to make theoretical rescue of the course of the undergraduate discipline Administration Applied to Nursing throughout the history of teaching of Nursing in Brazil, connecting it to the teaching of Costs. We noticed, inserted in the teaching of this discipline, many direct quotes related to the themes of Costs or Economics in Health, from the earliest curriculum in Nursing, dating back to 1890 , to the National Curricular Policies of the Nursing Undergraduate Course, of 2001, in addition to indirect quotes related to management, decision taking, and advising in health projects, among others, but with no continuity.

\section{KEY WORDS}

Nursing (organization and administration).

Costs and cost analysis. Teaching.

\section{RESUMEN}

Este texto tuvo como objetivo realizar un rescate teórico sobre la trayectoria de la disciplina de Administración Aplicada a la enfermería, en el pregrado, a lo largo de la historia de la enseñanza de la Enfermería Brasileña, relacionándola a la enseñanza de Costos. Percibimos, insertadas en la enseñanza de esa disciplina, diversas citaciones directas referentes al tema Costos o Economía en Salud, desde el primer currículo de Enfermería de 1890 hasta las Directivas Curriculares Nacionales del Pregrado en Enfermería del 2001, además de citaciones indirectas relacionadas al gerenciamiento, toma de decisión, asesorías en proyectos de salud, entre otras, pero sin ninguna continuidad.

\section{DESCRIPTORES}

Enfermería (organización y administración).

Costos y análisis de costo.

Enseñanza.

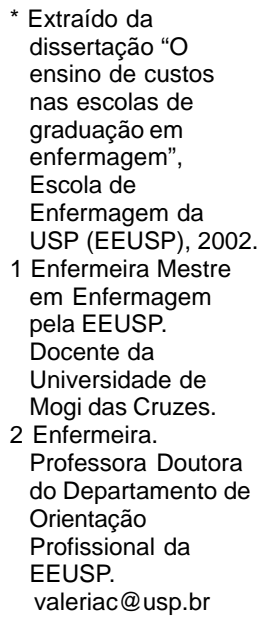




\section{INTRODUÇÃO}

O estudo de aspectos econômicos em saúde mostra-se extremamente atual e oportuno, uma vez que as organizações de saúde vivem momentos de grande competitividade, de busca pela qualidade do atendimento aos clientes e de necessidade de incorporação de tecnologia de ponta.

O crescimento exponencial dos custos em saúde está diretamente relacionado a uma série de fatores, tais como: 0 emprego de novas tecnologias; o aumento da expectativa de vida da população; o crescimento da demanda por atendimento, devido a universalização do acesso à saúde; a escassez de mão de obra qualificada, acarretando baixa produtividade; a má gestão das organizações, devido à incapacidade administrativa dos profissionais de saúde; a não implantação de sistemas de controle de custos; e os desperdícios na cadeia produtiva, entre outros ${ }^{(1-2)}$.

De acordo com o Conselho Internacional de Enfermagem $^{(3)}(\mathrm{ICN})$, todos os países compartilham de um problema comum, de custos galopantes na área de saúde frente a recursos ou orçamentos limitados.

Outras autoras ${ }^{(4)}$ comentam que a restrição de custos é responsabilidade de qualquer provedor de assistência à saúde, e a sobrevivência de muitas organizações depende de sua capacidade de gerir seus custos.

Anselmi $^{(5)}$ referindo-se aos enfermeiros afirma que

no momento atual em que o setor saúde passa por reformas estruturais e conjunturais significativas percebe-se fortes exigências à profissão para que assuma um papel efetivo na gestão econômica das instituições de saúde.

A Organização Mundial de Saúde aponta a enfermeira como o profissional da área de saúde com o maior potencial para assegurar uma assistência rentável, ou seja, eficaz em função dos $\operatorname{custos}^{(3)}$.

Isso vem ao encontro com o que alguns estudiosos das tendências mundiais afirmaram em 1993, que as enfermeiras podem ser responsáveis por 40 a $50 \%$ do faturamento dos hospitais ${ }^{(6)}$.

As enfermeiras constituem um nível decisório importante na alocação de recursos, quando decidem em suas unidades de trabalho, as prioridades de seus serviços, decidem quem prestará e quanto tempo será despendido nos cuidados, e quais recursos serão empregados ${ }^{(3)}$.

Atualmente, as enfermeiras administradoras estão cada vez mais sendo envolvidas em decisões financeiras e no planejamento orçamentário de suas instituições, tendo que gerir recursos (humanos, materiais e financeiros) muitas vezes $\operatorname{escassos}^{(3)}$.
Em relação à preocupação com o custo do atendimento à saúde, observa-se que os aspectos econômico-financeiros relativos à assistência de Enfermagem foram historicamente ignorados, apesar dos enfer-meiros efetivamente gerenciarem as unidades assistenciais, através do planejamento, coordenação, supervisão e controle do trabalho ${ }^{(7)}$.

Em 1984, apontava-se para a pequena quantidade de trabalhos publicados sobre custos da assistência de Enfermagem na literatura brasileira, acrescentando que esta matéria nem sequer era objeto de debate ou estudo nos currículos dos cursos de administração, mostrando não haver nos enfermeiros, ainda, uma consciência de custos hospitalares e da contribuição da sua equipe para redução ou adequação destes à eficácia do serviço ${ }^{(8)}$.

Comenta-se que a educação econômica em saúde deve ser iniciada na escola de Enfermagem, e que os instrutores e empregadores devem proporcionar aos enfermeiros os meio de adquirir responsabilidade fiscal, incluindo o planejamento orçamentário nos currículos de Enfermagem e em programas de administração ${ }^{(4)}$.

O profissional enfermeiro, engajado no processo gerencial das Instituições de Saúde, seja como Gerentes ou Diretores de Divisão, de Serviço, ou Chefes de Unidades, necessitam mais do que nunca buscar conhecimentos sobre custos, reconhecendo seu papel como agente de mudanças no alcance de resultados positivos, bem como buscando o equilíbrio entre quali dade, quantidade e recursos limitados.

Diante da gama de fatores responsáveis pelo aumento de custos em Saúde e pela importância da participação do enfermeiro como agente atuante nesse processo, há necessidade de se estudar a inserção desses conteúdos na graduação em Enfermagem.

Portanto o objetivo deste trabalho foi levantar a inserção do tema Custos no ensino da disciplina Administração Aplicada à Enfermagem.

\section{MÉTODO}

Trata-se de um estudo exploratório, descritivo e documental acerca das legislações pertinentes ao ensino da Graduação de Enfermagem.

A população foi constituída pelo conjunto de Leis, Decretos, Pareceres, Portarias e Resoluções do Ensino de Enfermagem, entre os anos de 1890 e 2001, cuja fonte foi o site do Ministério da Educação (www.mec.gov.br) e os livros: Enfermagem, legislação e assuntos $\operatorname{correlatos}^{(9)}$ e Legislação em Enfermagem: atos normativos do exercício e do ensino de Enfermagem ${ }^{(10)}$. 
Adotou-se o ano inicial de 1890, por corresponder à criação da primeira Escola de Enfermagem do Brasil e o ano de 2001 por ser o ano final da coleta de dados para esse estudo.

\section{RESULTADOSE DISCUSSÃO}

\section{Custos e o ensino da disciplina Administração Aplicada à Enfermagem}

A compreensão de qualquer área do conhecimento encontra-se intimamente relacionada com suas origens e raízes, fazendo-se necessário buscar na história explicações para fatos ocorridos na atualidade ${ }^{(11)}$.

A história do ensino da disciplina Administração Aplicada à Enfermagem no Brasil teve suas raízes nos currículos adotados pelas primeiras Escolas de Enfermagem do país, desde o fim do século XIX ${ }^{(12)}$.

Fazendo uma retrospectiva, verificou-se que a história da disciplina Administração em Enfermagem no Brasil iniciou-se a partir da criação, no Hospício Nacional de Alienados, da Escola Profissional de Enfermeiros e Enfermeiras criada pelo Decreto n. ${ }^{\circ} 791$ de 27 de setembro de $1890^{(13)}$.

A criação dessa escola tinha como objetivo preparar enfermeiros e enfermeiras para trabalharem nos hospícios e hospitais civis e militares, nos moldes da escola existente em Salpetrière, na França ${ }^{(14)}$. Posteriormente, a instituição recebeu a denominação de Escola de Enfermagem Alfredo Pinto e surgiram evidências, nessa escola, a respeito do primeiro programa de ensino de Enfermagem no Brasil, quando $\mathrm{o}$ artigo $2^{\circ}$ do Decreto n. ${ }^{\circ} 791$ de $1890^{(15)}$ determinou que o curso constaria de:

- noções práticas de propedêutica clínica;

- noções gerais de anatomia, fisiologia, higiene hospitalar, curativos, pequena cirurgia, cuidados especiais a certas categorias de enfermos e aplicações balneoterápicas;

- administração interna e escrituração do serviço sanitário e econômico das enfermarias (grifo nosso).

Constatou-se neste primeiro programa de ensino de Enfermagem no Brasil duas referências importantes, uma relativa ao ensino de Administração e outra da atividade de escrituração dos aspectos econômicos das enfermarias.

\section{Para Ferreira ${ }^{(16)}$ econômico refere-se à}

economia, ao controle de despesas e parcimônia nos gastos"; e economia está relacionada "à arte de bem administrar uma casa ou um estabelecimento particular ou público; contenção ou moderação nos gastos; ciência que trata dos fenômenos relativos à produção, distribuição, acumulação e consumo de bens materiais.

Dando continuidade aos fatos, algumas autoras ${ }^{(17)}$ relataram uma outra tentativa de organizar o ensino de Enferma- gem no Brasil, com a criação da Escola de Enfermagem do Departamento Nacional de Saúde Pública pelo Decreto n. ${ }^{\circ}$ 16.300 de 31 de dezembro de1923, então dirigido por Carlos Chagas, atual Escola Ana Nery. A partir da criação desta escola nasceu a Enfermagem moderna em nosso país, baseada na implantação do modelo Nightingale.

Para implantação e funcionamento dessa escola a Fundação Rockefeller enviou para o Brasil nove enfermeiras americanas, com o intuito de estruturar o Serviço de Enfermagem de Saúde Pública no Rio de Janeiro, sendo elas também as organizadoras e primeiras professoras da escola ${ }^{(11)}$.

A formação de novos enfermeiros foi reorientada para a atuação em Saúde Pública (pela necessidade de formar profissionais para garantir o saneamento dos portos, principalmente no Rio de Janeiro, em virtude do surgimento de constantes epidemias, sendo esse controle uma condição necessária à continuidade de importações e exportações), preservando-se no entanto, as disciplinas voltadas à administração hospitalar ${ }^{(12)}$.

O curso da Escola de Enfermeiras visava instrução teórica e prática, feitas simultaneamente em dois anos e quatro meses, divididos em cinco séries. As quatro primeiras séries constituíam a parte geral do curso e a última série seria destinada às especializações em Enfermagem Clínica, Enfermagem em Saúde Pública ou Administração Hospitalar ${ }^{(10)}$.

No Programa de instrução geral desse primeiro currículo brasileiro apareciam duas disciplinas voltadas para a administração: Administração Hospitalar e Serviço Administrativo Hospitalar e nenhuma disciplina de Administração Sanitária ou Administração de Saúde Pública ${ }^{(18)}$, apontando este fato para uma possível contradição, uma vez que naquele momento histórico a preocupação preponderante era com Saúde Pública, ou seja, com as questões de higiene da população através do saneamento das áreas urbanas e rurais, além de ações diretas no combate às endemias através da Educação Sanitária.

Observou-se que este primeiro currículo estava direcionado para o trabalho de Enfermagem em instituições hospitalares, atendendo à medicina curativa e hospitalar ${ }^{(14)}$.

Não se encontrou, no currículo brasileiro de 1923, nenhuma referência em relação a custos ou aspectos econômicos em saúde.

A Escola Ana Nery foi considerada instituição complementar da Universidade do Brasil conforme Lei n. ${ }^{\circ} 452$ de 05 de julho de 1937, passando a pertencer definitivamente à Universidade do Brasil pelo Decreto n. ${ }^{\circ} 21.321$ de 18 de junho de $1946^{(13)}$.

A respeito de novas mudanças curriculares, essas aconteceram em 1949, quando sofreram sua primeira reformulação ${ }^{(13)}$. 
O ensino da Enfermagem em 1949 passou a ser regulado pela Lei n. ${ }^{\circ} 775$ de 06 de agosto de $1949^{(19)}$, regulamentada pelo Decreto n. ${ }^{\circ} 27.426$ de 14 de novembro de 1949 que dispõe sobre o currículo dos cursos e as condições de preparação de enfermeiros, determinando a sua duração em 4 anos e tendo como pré-requisito a conclusão do curso colegial, sendo esse currículo uma adaptação do "Currículum Guide" norte americano de 1937.

Verificou-se nesse currículo um grande número de especialidades médicas, acompanhadas do conteúdo de Enfermagem, determinando uma maior fragmentação do ensino ${ }^{(20)}$. Nesse novo currículo foi incluída a disciplina Princípios de Administração Sanitária e excluídas as disciplinas de Administração voltadas para a área hospitalar, estando previstas apenas nos cursos de especialização.

Novamente percebeu-se uma contradição neste modelo curricular, uma vez que nesta época os novos estabelecimentos hospitalares, principalmente os de ensino, passaram a ser o principal mercado de trabalho.

Justificando a inclusão da disciplina Princípios de Administração Sanitária neste novo currículo, ela surgiu como uma resposta significativa frente àquele conjunto de fatos históricos ocorridos na década de 20, quando a preocupação era realmente com a Saúde Pública e o controle das endemias ${ }^{(20)}$.

Mesmo não estando presente neste currículo de graduação, disciplinas de Administração voltadas para a área hospitalar, no programa de ensino proposto pelo Decreto $\mathrm{n}^{\circ} 27.426$ de 14 de novembro de 1949, encontrou-se no seu artigo $5^{\circ}$ a inclusão do tema "Economia Hospitalar", a ser administrado já na primeira série ${ }^{(21)}$.

Essa foi a segunda referência sobre aspectos econômicos, que envolvem custos, nos programas de ensino de Enfermagem.

Em 1961, com a Lei n. ${ }^{\circ} 2.604$ de 1955, regulamentada pelo Decreto n. ${ }^{\circ} 50.387$ de 1961, que dispõe sobre o exercício da Enfermagem, deu-se à enfermeira o poder de mando em relação aos auxiliares e atendentes de enfermagem e aos cuidados de Enfermagem ${ }^{(17)}$. Ocorreu nesse momento a passagem definitiva dos cursos de Enfermagem para o nível superior, em obediência aos disposto na Lei n. ${ }^{\circ} 2.995$ de 1956.

Em 1962 o Conselho Federal de Educação - CFE ${ }^{(22)}$, através do Parecer n. ${ }^{\circ} 271$ de 19 de outubro de 1962 fixou o currículo mínimo para o Curso de Enfermagem, determinando sua duração em três anos para o curso geral, que incluía as disciplinas de Fundamentos de Enfermagem, Enfermagem Médica, Enfermagem Cirúrgica, Enfermagem Psiquiátrica, Enfermagem Obstétrica e Ginecológica, Enfermagem Pediátrica, Ética e História da Enfermagem e Administração.
Nesta reestruturação a disciplina de Administração voltou-se para a área hospitalar, sendo reintegrada ao elenco de disciplinas do curso de Enfermagem, passando a fazer parte também das especializações específicas neste currículo.

Não obstante houvesse o retorno da disciplina de Administração ao currículo, não foi encontrado no Parecer n. ${ }^{\circ} 271$ de 19 de outubro de 1962 do CFE nenhuma referência a "custos" ou aspectos "econômicos" em saúde.

Prosseguindo até o início da década de 70, ocorre a reforma universitária e é aprovado o Parecer CFE n. ${ }^{\circ} 163$ de 28 de janeiro de 1972, definindo como carga horária mínima curricular dos cursos de Enfermagem e Obstetrícia 2.500 horas $^{(23)}$.

Esse Parecer também estabeleceu que os cursos de Enfermagem e Obstetrícia compreenderiam três partes sucessivas: pré-profissional; tronco profissional comum levando à graduação do enfermeiro e habilitações, conduzindo à formação do Enfermeiro Médico-Cirúrgico, da Enfermeira Obstétrica ou Obstetriz e do Enfermeiro de Saúde Pública ${ }^{(23)}$.

Neste momento o número de disciplinas de Administração elevou-se para cinco, uma vez que este conteúdo foi incorporado nas habilitações em Enfermagem Obstétrica, Enfermagem Médico-Cirúrgica e Enfermagem em Saúde Pública.

Pode-se elencar essas cinco disciplinas da seguinte maneira:

1. Administração Aplicada à Enfermagem, no tronco profissional comum

2. Administração de Centro Cirúrgico e 3. Administração de Serviço de Enfermagem Hospitalar, ambas na Habilitação em Enfermagem Médico-cirúrgica.

4. Administração de Serviços de Enfermagem em Maternidades e Dispensários Pré-natais, na Habilitação em Enfermagem Obstétrica.

5. Administração de Serviços de Enfermagem em Unidades de Saúde, na Habilitação em Enfermagem de Saúde Pública.

Neste currículo de 1972 notou-se uma valorização das especializações na graduação e a busca de uma maior intelectualização do saber administrativo, que se efetivou nos cursos de pós-graduação em Enfermagem a partir de 1972, quando foi criado o primeiro curso em nível de mestrado $^{(20)}$.

Não foi encontrado no Parecer n. ${ }^{\circ} 163$ de 1972 do CFE nenhuma referência a "custos" ou aspectos "econômicos" em saúde. 
Dando prosseguimento à retrospectiva do ensino de Administração em Enfermagem, no ano de 1986 foi aprovada a nova Lei do Exercício Profissional em Enfermagem, Lei n. ${ }^{\circ} 7.498$ de 25 de julho de 1986.

Essa Lei evidenciava a importância do ensino da disciplina Administração Aplicada à Enfermagem na formação do profissional, uma vez que colocou como função privativa do enfermeiro em seu enunciado, entre outras atividades: direção do órgão de Enfermagem integrante da estrutura básica da instituição de saúde, pública e privada, e chefia de serviço e de unidade de Enfermagem; organização e direção dos serviços de Enfermagem e de suas atividades técnicas e auxiliares nas empresas prestadoras desses serviços; o planejamento, organização, coordenação, orientação e avaliação dos serviços de assistência de Enfermagem, bem como a participação nos pro-gramas de treinamento e aprimoramento do pessoal de saúde, particularmente nos programas de educação continuada ${ }^{(24)}$.

Também não se encontrou na Lei do Exercício Profissional em Enfermagem, Lei n. 7.498 de 1986, aspectos relacionados à "custos" ou aspectos "econômicos" em saúde, porém o enfoque administrativo que foi dado à função privativa do enfermeiro pode vir a indicar a necessidade desses conhecimentos no gerenciamento de Instituições de Saúde públicas e privadas.

Algumas autoras ${ }^{(12,25)}$ teceram comentários a respeito de mudanças no ensino da Administração em Enfermagem, relembrando a realização de cinco encontros nacionais na década de 80, denominados Ciclos de Estudos de Professores de Administração Aplicada à Enfermagem, cuja proposta nos anos de 1985 a 1989 foi repensar coletivamente o ensino da disciplina. Como resultado desses encontros destacaram-se as recomendações de incorporar ao conteúdo da disciplina o referencial político, econômico (grifo nosso) e cultural no ensino da Administração.

Percebe-se aqui a preocupação com a inserção de aspectos "econômicos" no ensino da Administração, mas sem a explicitação de seu conteúdo.

Em 1994 foi aprovada a Portaria MEC n. ${ }^{\circ} 1.721$ de 15 de dezembro de 1994 regulamentando o ensino dos Cursos de Graduação em Enfermagem, passando o currículo a obedecer uma carga horária de 3.500 horas e duração mínima de 4 anos. Nesta Portaria a disciplina de Administração em Enfermagem passou a abranger 15\% da carga horária total do curso, correspondendo a 525 horas $^{(26)}$.

Esse foi um momento significativo tanto para a Enfermagem como para a Administração em Enfermagem, uma vez que essa Portaria evidenciou o reconhecimento e a importância dessa disciplina na formação do enfermeiro ${ }^{(27)}$.
Não encontrou-se na Portaria supra citada aspectos relativos a "Custos" ou "econômicos" inseridos nos conteúdos curriculares mínimos do Curso de Graduação em Enfermagem.

Faz-se necessário ressaltar que, em virtude da nova Lei de Diretrizes e Bases da Educação Nacional - Lei n. ${ }^{\circ} 9.394$ promulgada em 20 de dezembro de 1996, foi assegurada às instituições de ensino superior a autonomia didático-científica, porém foi recomendado o fim dos currículos mínimos e a adoção de diretrizes curriculares para todos os cursos de graduação como veremos a seguir ${ }^{(28)}$.

No ano de 2000 o Ministério da Educação através da Portaria n. $^{\circ} 1518$ de 14 de junho de 2000, constituiu entre outras uma Comissão de Especialistas de Ensino de Enfermagem para estabelecer as Diretrizes Curriculares do Curso de Graduação em Enfermagem ${ }^{(29)}$. Essa Comissão de Especialistas definiu o perfil, as competências e habilidades do bacharel em Enfermagem.

Quanto ao perfil, sugere que o enfermeiro deve possuir competências técnocientíficas, ético-políticas, sócio-educativas que permitam comprometer-se com os investimentos (grifo nosso) voltados para a solução de problemas sociais, no entanto, não fica claro se o sentido desse termo está relacionado a investimentos econômicos.

Prosseguindo, em 2001 o Conselho Nacional de Educação, através da Câmara de Educação Superior, instituiu as Diretrizes Curriculares Nacionais do Curso de Graduação em Enfermagem pela Resolução CNE/CES n. ${ }^{\circ} 3$ de 07 de novembro de $2001^{(30)}$.

Tal Resolução define os princípios, fundamentos, condições e procedimentos da formação de enfermeiros para aplicação em âmbito nacional na organização, desenvolvimento e avaliação dos projetos pedagógicos dos Cursos de Graduação em Enfermagem.

Ao analisar-se o texto, encontrou-se entre as Competências e Habilidades gerais propostas:

I - Tomada de decisões: o trabalho dos profissionais de saúde deve estar fundamentado na capacidade de tomar decisões visando o uso apropriado, eficácia e custo efetividade (grifo nosso), da força de trabalho, de medicamentos, de equipamentos, de procedimentos e de práticas. Para este fim, os mesmos devem possuir competências e habilidades para avaliar, sistematizar e decidir as condutas mais adequadas, baseadas em evidências científicas;

II - Administração e gerenciamento: os profissionais devem estar aptos a tomar iniciativas, fazer o gerenciamento e administração tanto da força de trabalho quanto dos re- 
cursos físicos, materiais e de informação, da mesma forma que devem estar aptos a serem empreendedores, gestores, empregadores ou lideranças na equipe de saúde;

Encontrou-se entre as Competências e Habilidades específicas propostas:

XXIII - interferir na dinâmica de trabalho institucional, reconhecendo-se como agente desse processo;

XXX - participar da composição das estruturas consultivas e deliberativas do sistema de saúde;

XXXI - assessorar órgãos, empresas e instituições em projetos de saúde;

Pode-se perceber que, além do enfoque direto da abordagem de Custos, ao citar o custo efetividade (a efetividade é medida em relação ao resultado alcançado, o custo efetividade consiste resumidamente, na adoção de estratégias capazes de alcançar o melhor resultado ao menor custo possível de ser assumido), encontrou-se também indícios da necessidade do conhecimento de "Custos" para plena atuação do enfermeiro nas demais competências e habilidades supra citadas, pois para ser capaz de tomar decisões, gerenciar, opinar, deliberar e assessorar o enfermeiro deve conhecer todas as variáveis envolvidas nos processos, e sem dúvida a variável econômica ou referente a Custos em Saúde deve ser considerada.

Entre os conteúdos essenciais para o curso de Graduação em Enfermagem citados na Resolução CNE/CES n. ${ }^{\circ} 3$ de 2001, está incluída a disciplina Administração em Enfermagem, com seus conteúdos teóricos e práticos da administração do processo de trabalho de Enfermagem e da assistência de Enfermagem.

Em relação aos conteúdos programáticos da disciplina de Administração Aplicada à Enfermagem, encontrou-se dois trabalhos que fazem referências ao ensino de "Custos".

O primeiro deles feito por Gomes ${ }^{(20)}$, no final da década de 80 sobre "Administração em Enfermagem: Constituição Histórico-Social do Conhecimento", identificou nos conteúdos programáticos das disciplinas de Administração de 48 Cursos de Graduação em todo o país, seis escolas que abordavam temas/conteúdos relativos a economia, compreendendo finanças, custos e orçamentos (grifo nosso).

\section{REFERÊNCIAS}

(1) Medici AC. Financiamento e contenção de custos nas políticas de saúde: tendências atuais e perspectivas futuras. Planej Polít Públicas. 1990;(4):83-93.

(2) Medici AC, Marques RM. Sistema de custos como instrumento de eficiência e qualidade dos Serviços de Saúde. Cad Fundap. 1996;16(19):47-59.
O segundo trabalho escrito por Nimtz ${ }^{(13)}$ no final da década de 90 sobre o ensino da disciplina de Administração em Enfermagem em 10 escolas de Graduação da Grande São Paulo, ao levantar os objetivos gerais e objetivos específicos dessa disciplina, encontrou em três escolas referências diretas ao conhecimento e administração de recursos financeiros (grifo nosso).

\section{CONSIDERAÇÕES FINAIS}

Diante desse levantamento, pode-se compreender que o ensino da disciplina de Administração em Enfermagem foi conquistando, gradativamente, importância na formação do enfermeiro, sendo de fato essencial como instrumento de trabalho no gerenciamento de Serviços de Saúde, Recursos Humanos, Recursos Materiais, Administração da Assistência de Enfermagem e Educação Continuada.

Percebe-se também, inseridas no ensino dessa disciplina, diversas citações diretas referentes ao tema Custos ou Economia em Saúde desde o primeiro currículo de Enfermagem de 1890 até as Diretrizes Curri-culares Nacionais do Curso de Graduação em Enfermagem de 2001, relembrando-as: serviço econômico das enfermarias, economia hospitalar, referencial econômico, comprometimento com investimentos, custo efetividade, finanças, custos, orçamentos, conhecimento e administração de recursos financeiros; além das citações indiretas relacionadas ao gerenciamento, tomada de decisão, assessorias em projetos de saúde, entre outras.

Diante da atual complexidade econômico-administrativa em todos os setores, inclusive na Saúde, considera-se importante que as Escolas de Graduação ensinem conteúdos específicos sobre esse tema, com a finalidade de aproximar o aluno de graduação em relação a conteúdos relacionados a aspectos econômicos e ao gerenciamento de Custos nos Serviços de Enfermagem.

O enfermeiro deverá estar apto à atender as demandas das Organizações de Saúde, preocupadas com a elevação crescente de custos, a racionalização de recursos, o controle de desperdícios e a otimização de resultados, assumindo assim um papel efetivo na gestão econômica dos Serviços de Enfermagem e, conseqüentemente, das Instituições de Saúde.

(3) Consejo Internacional de Enfermeras (CIE). La calidad, los costos y la enfermeria. [Presentado en el Dia Internacional de La Enfermera; 1993 mayo 12; Geneva].

(4) Marquis BL, Huston CJ. Administração e liderança em enfermagem: teoria e aplicação. $2^{\mathrm{a}}$ ed. Porto Alegre: Artes Médicas; 1999. 
(5) Anselmi ML. Quadro de referência para elaboração do orçamento de enfermagem em Instituições Hospita-lares. [tese] Ribeirão Preto: Escola de Enfermagem de Ribeirão Preto/USP; 2000.

(6) Aburdene P, Naisbitt J. Megatendências para as mulheres. Rio de Janeiro: Rosa dos Tempos; 1993.

(7) Anselmi ML, Nakao JRS. A Enfermagem no processo de gestão econômica dos serviços de saúde: limites e possibilidades. Rev Bras Enferm. 1999;52(2):223-32.

(8) Almeida MH. Custos hospitalares na Enfermagem. Rio de Janeiro: Cultura Médica; 1984.

(9) Brasil. Ministério da Saúde. Fundação Serviços de Saúde Pública. Enfermagem, legislação e assuntos correlatos. $3^{\mathrm{a}}$ ed. Rio de Janeiro; 1974. 3v.

(10) Santos EF, Santos EB, Santana GO, Assis MF, Meneses RO. Legislação em enfermagem: atos normativos do exercício e do ensino de enfermagem. São Paulo: Atheneu; 1997.

(11) Germano RM. Educação e ideologia da enfermagem no Brasil. $2^{a}$ ed. São Paulo: Cortez; 1985.

(12) Sanna MC. A evolução da disciplina de administração aplicada à enfermagem na Escola de Enfermagem da USP no período de 1980 a 1995. Rev Esc Enferm USP. 1999;33(1):17-30.

(13) Nimtz MA. O ensino da disciplina de administração em enfermagem nas escolas de graduação da grande São Paulo. [dissertação] São Paulo: Escola de Enfermagem da USP; 1999.

(14) Galleguillos TGB. A inserção da saúde coletiva nos currículos dos cursos de graduação de escolas de enfermagem do Estado de São Paulo. [dissertação] São Paulo: Escola de Enfermagem da USP; 2001.

(15) Brasil. Decreto n. 791, de 27 de setembro de 1890. Cria no Hospício Nacional de Alienados uma escola profissional de enfermeiros e enfermeiras. In : Brasil. Ministério da Saúde. Fundação Serviços de Saúde Pública. Enfermagem, legislação e assuntos correlatos. $3^{\text {a }}$ ed. Rio de Janeiro; 1974. v. 1 , p. $26-7$.

(16) Ferreira $\mathrm{ABH}$. Novo dicionário da língua portuguesa. $2^{\mathrm{a}} \mathrm{ed}$. Rio de Janeiro: Nova Fronteira; 1986

(17) Ciampone MHT, Leite MMJ, Gaidzinski RR. Ensino da disciplina administração em enfermagem: em busca de um novo paradigma. Rev Esc Enferm USP. 1996;30(n. esp):45-58.

(18) Brasil. Decreto n. 16.300, de 31 de dezembro de 1923. Aprova o regulamento do Departamento Nacional de Saúde Pública. In : Brasil. Ministério da Saúde. Fundação Serviços de Saúde Pública. Enfermagem, legislação e assuntos correlatos. $3^{\mathrm{a}} \mathrm{ed}$. Rio de Janeiro; 1974. v. 1, p. 57-66.

(19) Brasil. Lei n. 775, de 6 de agosto de 1949. Dispõe sobre o ensino de enfermagem no país e dá outras providências. In : Brasil. Ministério da Saúde. Fundação Serviços de Saúde Pública. Enfermagem, legislação e assuntos correlatos. $3^{\mathrm{a}}$ ed. Rio de Janeiro; 1974. v. 1, p. 154-7.
(20) Gomes ERG. Administração em enfermagem: constituição histórico social do conhecimento. [tese] Ribeirão Preto: Escola de Enfermagem de Ribeirão Preto/USP, 1991.

(21) Brasil. Decreto n. 27.426, de 14 de novembro de 1949. Aprova o regulamento básico para os cursos de enfermagem e de auxiliar de enfermagem. In : Brasil. Ministério da Saúde. Fundação Serviços de Saúde Pública. Enfermagem, legislação e assuntos correlatos. $3^{\mathrm{a}}$ ed. Rio de Janeiro; 1974 v. 1 , p. $161-72$

(22) Brasil. Parecer n. 271/62, de 19 de outubro de 1962. Dispõe sobre o currículo mínimo do curso de enfermagem. In : Brasil. Ministério da Saúde. Fundação Serviços de Saúde Pública. Enfermagem,legislação e assuntos correlatos. $3^{\mathrm{a}} \mathrm{ed}$. Rio de Janeiro; 1974. v. 2, p. 249-53.

(23) Brasil. Parecer n. 163/72, C.C.R. de Currículos, de 28 de janeiro de 1972. Dispõe sobre o currículo mínimo dos cursos de enfermagem e obstetrícia. In : Brasil. Ministério da Saúde. Fundação Serviços de Saúde Pública. Enfermagem, legislação e assuntos correlatos. $3^{\mathrm{a}}$ ed. Rio de Janeiro; 1974. v. 3 , p. 721-4.

(24) Brasil. Lei n. 7.498, de 25 de julho de 1986. Dispõe sobre a regulamentação do exercício da enfermagem e dá outras providências. In: Conselho Regional de Enfermagem - COREN-SP. Documentos básicos de enfermagem. São Paulo; 2001.

(25) Leite MMJ. O ensino da disciplina administração aplicada à enfermagem: compreensão das graduandas. [tese] São Paulo: Faculdade de Educação da USP; 1994.

(26) Brasil. Portaria n. 1721, de 15 de dezembro de 1994. Fixa os conteúdos mínimos e duração do curso de graduação em enfermagem. Diário Oficial da República Federativa do Brasil, Brasília, 16 dez. 1994. Seção 1, p. 19801-2.

(27) Leite MMJ. Tecnologias educacionais: possibilidades e dificuldades no ensino de administração em enfermagem. [tese] São Paulo: Escola de Enfermagem da USP; 2000.

(28) Xavier IM, Fernandes JD, Cappo Bianco MHB, Ceribelli MIPF. Diretrizes Curriculares Nacionais para o curso de graduação em enfermagem. Rev SOBECC. 2001;6(4):8-9.

(29) Brasil. Ministério da Educação. Secretaria de Educação Superior. Departamento de Políticas de Ensino Superior. Comissão de Especialistas de Ensino de Enfermagem. Portaria n. 1.518 , de 14 de junho de 2000. Curso de graduação em enfermagem: Diretrizes Curriculares. [online] Disponível em:www.enfvirtual.hpg.ig.com.br/leis/diretrizes.htm (Acesso em 23 mar. 2002).

(30) Conselho Nacional de Educação. Câmara de Educação Superior. Resolução CNE/CES n. 3, de 7 de novembro de 2001. Institui Diretrizes Curriculares Nacionais do curso de graduação em enfermagem. [online] Disponível em: www.mec.gov.br/ cne/ftp/CES/CES03.doc (Acesso em 23 mar. 2002). 